\title{
FIRST CCD OBSERVATIONS OF MAGELLANIC CLOUD VARIABLE STARS FROM THE MT JOHN UNIVERSITY OBSERVATORY, NEW ZEALAND
}

\author{
WILLIAM TOBIN, A.C. GILMORE, ALAN WADSWORTH, S.R.D. WEST \\ Department of Physics and Mt John University Observatory, \\ University of Canterbury, Christchurch 1 \\ New Zealand
}

Late in 1988 the Mt John University Observatory acquired a cryogenic CCD system from Photometrics Ltd (Tucson). The chip is a Thomson CSF TH7882 CDA comprising $384 \times 576$ pixels. As part of the evaluation process, we have begun two differential photometry programs of the Magellanic Clouds using the Mt John 0.6m Boller \& Chivens telescope. On this telescope each CCD pixel corresponds to 0.6 arcsec. Mt John's southerly latitude $\left(44^{\circ} \mathrm{S}\right)$ permits year-round observations of the Clouds.

The first program concerns B, V and I photometry of five blue eclipsing binaries selected, on the basis of Gaposchkin's $(1970,1977)$ photographic light curves, to have roughly equal components with minimal interaction. HV 12634 has also been observed for comparison with the CCD light curves published by Jensen et al. (1988). Fig. 1 shows the B observations so far obtained for HV 1761, but the reduction is preliminary, being based on aperture-integrated magnitudes. The field is populous, and a final reduction will require use of a crowded-field reduction package such as ROMAFOT.

The second program involves a search for variable stars down to $\mathrm{V} \sim 15$ in the ESO Magellanic Cloud 'Key Programme' fields around NGC330 in the Small Magellanic Cloud (SMC), and westwards from NGC1978 in the Large Magellanic Cloud (LMC). Reduction of these images also awaits implementation in Christchurch of a satisfactory crowded-field reduction package.

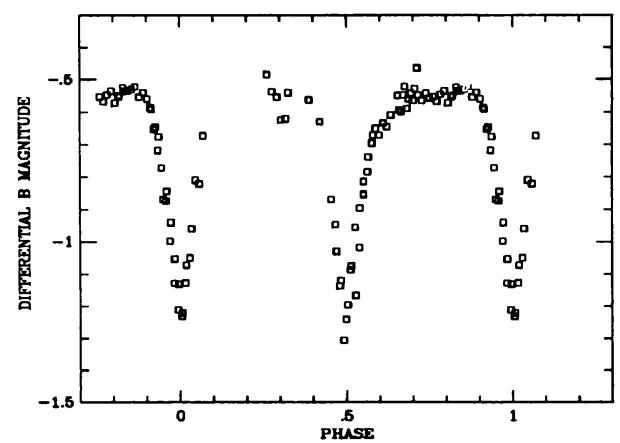

Figure 1. Preliminary B photometry of the eclipsing binary HV 1761 in the SMC (Period=1.47 day).

\section{References}

Jensen, K.S., Clausen, J.V. Giménez, A. (1988), Astron. Astrophys. Suppl. Ser. 74, 331.

Gaposchkin, S. (1970), SAO Special Report No. 310.

Gaposchkin, S. (1977), SAO Special Report No. 380. 